\title{
The Elementary Process of Bremsstrahlung
}




\section{World Scientific Lecture Notes in Physics}

\section{Published}

Vol. 49: Field Theory, Disorder and Simulations

G Parisi

Vol. 50: The Quark Structure of Matter $M$ Jacob

Vol. 51: Selected Topics on the General Properties of Quantum Field Theory $F$ Strocchi

Vol. 52: Field Theory: A Path Integral Approach

A Das

Vol. 53: Introduction to Nonlinear Dynamics for Physicists HD I Abarbanel, et al.

Vol. 54: Introduction to the Theory of Spin Glasses and Neural Networks $\checkmark$ Dotsenko

Vol. 55: Lectures in Particle Physics $D$ Green

Vol. 56: Chaos and Gauge Field Theory TS Biro, et al.

Vol. 57: Foundations of Quantum Chromodynamics (2nd ed.) TMuta

Vol. 59: Lattice Gauge Theories: An Introduction (2nd ed.) HJRothe

Vol. 60: Massive Neutrinos in Physics and Astrophysics $R$ N Mohapatra and $P B P a l$

Vol. 61: Modern Differential Geometry for Physicists (2nd ed.) C J Isham

Vol. 62: ITEP Lectures on Particle Physics and Field Theory (In 2 Volumes) MA Shifman

Vol. 64: Fluctuations and Localization in Mesoscopic Electron Systems $M$ Janssen

Vol. 65: Universal Fluctuations: The Phenomenology of Hadronic Matter $R$ Botet and M Ploszajczak

Vol. 66: Microcanonical Thermodynamics: Phase Transitions in "Small" Systems D HE Gross

Vol. 67: Quantum Scaling in Many-Body Systems MA Continentino

Vol. 69: Deparametrization and Path Integral Quantization of Cosmological Models C Simeone

Vol. 70: Noise Sustained Patterns Markus Loecher

Vol. 73: The Elmentary Process of Bremsstrahlung W. Nakel and E. Haug 
World Scientific Lecture Notes in Physics - Vol. 73

\section{The Elementary Process of Bremsstrahlung}

\section{Eberhard Haug}

Institut für Astronomie und Astrophysik

Universität Tübingen, Germany

\section{Werner Nakel}

Physikalisches Institut

Universität Tübingen, Germany 


\section{Published by}

World Scientific Publishing Co. Pte. Ltd.

5 Toh Tuck Link, Singapore 596224

USA office: Suite 202, 1060 Main Street, River Edge, NJ 07661

UK office: 57 Shelton Street, Covent Garden, London WC2H 9HE

\section{British Library Cataloguing-in-Publication Data}

A catalogue record for this book is available from the British Library.

\section{THE ELEMENTARY PROCESS OF BREMSSTRAHLUNG}

Copyright $\odot 2004$ by World Scientific Publishing Co. Pte. Ltd.

All rights reserved. This book, or parts thereof, may not be reproduced in any form or by any means, electronic or mechanical, including photocopying, recording or any information storage and retrieval system now known or to be invented, without written permission from the Publisher.

For photocopying of material in this volume, please pay a copying fee through the Copyright Clearance Center, Inc., 222 Rosewood Drive, Danvers, MA 01923, USA. In this case permission to photocopy is not required from the publisher.

ISBN $\quad 981-238-578-9$ 


\section{Preface}

This book is based on a review article about the elementary process of bremsstrahlung [W. Nakel, Phys. Rep. 243 (1994) 317]. When the author was encouraged by the editors of World Scientific to expand the review article to a lecture notes volume, he asked a colleague (E.H.) who has been working on the theory of bremsstrahlung, to be the co-author.

The aim of the book is to represent the theoretical and experimental developments in the elementary bremsstrahlung process, i.e., the triply differential cross section, and the status of the comparison and extent of agreement between them. It will be shown how far theory proceeded in the effort to describe the coupling of the radiation field to the electronatom system. Although the main importance is attached to electron-atom bremsstrahlung, we also discuss electron-electron bremsstrahlung, electronpositron bremsstrahlung, and further bremsstrahlung processes.

The book is mainly addressed to graduate students and is therefore endowed with classical and semiclassical considerations on the bremsstrahlung process and with problems to be solved. But it will also be of interest to physicists who are actively engaged in research since it summarizes and discusses the extensive theoretical work in the original literature. Last but not least, the book is addressed to those readers who may want to get a more general view on this subject since bremsstrahlung is related to the fundamentals of theory and appears in many branches of physics and in technical applications.

The authors are indebted to Prof. H. Ruder, Prof. G.J. Wagner, and Dr. H. Lindel for their support of the work, and to Prof. P. Grabmayr for his valuable help in the preparation of this book. We are very grateful to 
Prof. L.C. Maximon for helpful discussions. We wish to thank Dipl.-Phys. E. Gaertig, F. Engeser and U. Vogl for their extensive technical help in getting the manuscript into its final form.

We acknowledge the permission by Elsevier Science to reproduce the following figures from the article which appeared in Physics Reports [86]: Figs. 4.1, 4.2, 4.4, 4.5, 4.7 to $4.14,4.16,5.6$, and 6.1 to 6.6 .

Eberhard Haug Werner Nakel

Universität Tübingen, September 2003

\section{Note from the authors}

The authors would be grateful if the readers would inform them about any errors which they may find. Corrections for this book which come to the authors' attention will be posted on the World Wide Web at http://www.pit.physik.uni-tuebingen.de/nakel/nakel_ee.html but can also be obtained by writing to the authors. The contact e-mail address is nakel@pit.physik.uni-tuebingen.de. 


\section{Contents}

Preface $\quad v$

Chapter 1. Introduction 1

1.1 General introduction . . . . . . . . . . . . . . . . 1

1.2 Short historical note . . . . . . . . . . . . 4

1.3 Notations and definitions $\ldots \ldots \ldots \ldots$

Chapter 2. Classical and semiclassical considerations on the bremsstrahlung process $\quad 9$

2.1 Electron-nucleus bremsstrahlung . . . . . . . . . . . . . 9

2.1.1 Screening effects . . . . . . . . . . . . . . 16

2.1.2 Bremsstrahlung linear polarization . . . . . . . . 18

2.1.3 Bremsstrahlung from transversely polarized electrons . 22

2.2 Electron-electron bremsstrahlung . . . . . . . . . . . 23

2.3 Weizsäcker-Williams method of virtual quanta $\ldots \ldots \ldots 26$

Chapter 3. Theory of the elementary process of electron$\begin{array}{ll}\text { nucleus bremsstrahlung } & 27\end{array}$

3.1 Introduction . . . . . . . . . . . . . . . . . . 27

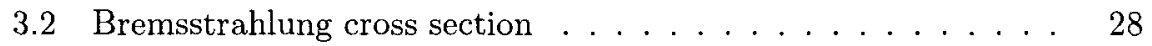

3.3 Born approximation (Bethe-Heitler formula) . . . . . . . . . 32

3.3 .1 Atomic screening . . . . . . . . . . . . . 47

3.3 .2 Nuclear recoil effects . . . . . . . . . . . . . . 50

3.3 .3 Effects of nuclear structure . . . . . . . . . . 51

3.4 Approximations with the Sommerfeld-Maue wave function . . . 55 
3.4.1 Sommerfeld-Maue wave function . . . . . . . . . 55

3.4 .2 Spin formalism . . . . . . . . . . . . . 61

3.4 .3 Cross section . . . . . . . . . . . . . . 63

3.4.4 Nonrelativistic approximation . . . . . . . . . . . 71

3.4.5 The short-wavelength limit . . . . . . . . . . 72

3.4.6 Approximation for the long-wavelength limit and for high electron energies . . . . . . . . . . . . . 74

3.4.7 Cross section in second Born approximation . . . . . . 76

3.5 Calculation using relativistic partial-wave expansions . . . . . 79

3.5.1 Partial wave expansion . . . . . . . . . . 79

3.5.2 Calculations with a relativistic self-consistent-field potential . . . . . . . . . . . . . . 89

3.5.3 Calculation for a pure Coulomb potential . . . . . . . 93

$\begin{array}{lll}3.6 & \text { Spin-dependent cross section and bremsstrahlung asymmetry . } & 97\end{array}$

3.7 Bremsstrahlung polarization . . . . . . . . . . . . . 104

3.7.1 General polarization correlation. . . . . . . . . . 104

3.7.2 Linear polarization in Born approximation . . . . . . 108

3.7.3 Circular polarization from polarized electrons in Born approximation . . . . . . . . . . . . . 112

3.8 Radiative corrections to bremsstrahlung . . . . . . . . 115

Chapter 4. Experiments on the elementary process of electron-nucleus bremsstrahlung

4.1 Survey of experimental devices . . . . . . . . . . . . . 119

4.2 Electron-photon coincidence experiments without regard to polarization variables . . . . . . . . . . . . . . . 121

4.2.1 Angular distributions of photons for fixed directions of outgoing electrons . . . . . . . . . . . . . 121

4.2.2 Angular distributions of electrons for fixed photon directions . . . . . . . . . . . . . . 123

4.2.3 Energy distributions for fixed electron and photon directions . . . . . . . . . . . . . . 125

4.2.4 Wide-angle bremsstrahlung experiments at very high energies . . . . . . . . . . . . . . 127

4.3 Electron-photon coincidence experiments including polarization variables . . . . . . . . . . . . . . . . . . . 128

4.3.1 Linear polarization of bremsstrahlung emitted by unpolarized electrons . . . . . . . . . . . . . . . . 128 
4.3.2 Photon emission asymmetry of bremsstrahlung from transversely polarized electrons . . . . . . . . . . 136

4.3.3 Further polarization correlations . . . . . . . . . . 141

4.4 Tagged photons . . . . . . . . . . . . . . . . . . . 143

Chapter 5. Theory of the elementary process of electron-electron bremsstrahlung $\quad 147$

5.1 Introduction . . . . . . . . . . . . . . . . . 147

5.2 Kinematics . . . . . . . . . . . . . . . . . . . 148

5.2 .1 Center-of-mass system . . . . . . . . . . . 151

5.2 .2 Laboratory system . . . . . . . . . . . . . . . 152

5.3 Cross section . . . . . . . . . . . . . . . 157

5.3 .1 Laboratory system . . . . . . . . . . . . . . . . . 161

5.3 .2 Center-of-mass system . . . . . . . . . . . . . . . 162

5.3.3 Center-of-mass system of the outgoing electrons . . . 162

5.4 Bremsstrahlung in the field of bound electrons . . . . . . 165

Chapter 6. Experiments on the elementary process of electron-electron bremsstrahlung

6.1 Electron-photon coincidence experiments without regard to polarization variables . . . . . . . . . . . . . 175

6.1.1 Angular distribution of photons for fixed electron direction . . . . . . . . . . . 176

6.1 .2 Energy distributions . . . . . . . . . . . . 178

6.2 Electron-photon coincidence experiments including polarization variables . . . . . . . . . . . . . . . . . . . 182

6.2.1 Linear polarization of electron-electron bremsstrahlung emitted by unpolarized electrons . . . . . . . . . . . 182

6.2.2 Further polarization correlations . . . . . . . . 185

Chapter 7. Some remarks on integrated cross sections and $\begin{array}{lr}\text { further bremsstrahlung processes } & 187\end{array}$

7.1 Integrated cross sections . . . . . . . . . . . . . . 187

7.2 Positron-nucleus bremsstrahlung . . . . . . . . . . . 190

7.3 Electron-positron bremsstrahlung . . . . . . . . . . . . . . 192

7.4 Two-photon bremsstrahlung . . . . . . . . . . . 196

7.5 Polarization bremsstrahlung . . . . . . . . . . . . . 203

7.6 Crystalline targets: coherent bremsstrahlung . . . . . . 206 
7.7 Bremsstrahlung from heavy particles . . . . . . . . 206

7.8 Bremsstrahlung in nuclear decays . . . . . . . . . . 207

7.8.1 Bremsstrahlung in $\beta$ decay . . . . . . . . . 207

7.8.2 Bremsstrahlung in orbital-electron capture . . . . . 207

7.8 .3 Bremsstrahlung in $\alpha$ decay . . . . . . . . 207

7.9 Bremsstrahlung in magnetic fields . . . . . . . . . . 208

7.9.1 Electron-nucleus bremsstrahlung in strong magnetic

fields . . . . . . . . . . . . . . . . 208

7.9.2 Synchrotron radiation (magnetobremsstrahlung) $\ldots 210$

7.10 Stimulated bremsstrahlung . . . . . . . . . . 210

$\begin{array}{ll}\text { Conclusion } & 211\end{array}$

$\begin{array}{lll}\text { Appendix A Problems } & 213\end{array}$

Appendix B Squared matrix element of electron-electron bremsstrahlung 241

$\begin{array}{ll}\text { Bibliography } & 249\end{array}$

Index 257 